\title{
Electromagnetic Fields from Dental Devices and their Effects on Human Health
}

\author{
Takashi Kameda ${ }^{1 *}$ and Kazuo Ohkuma ${ }^{2}$
}

${ }^{1}$ Department of Orthodontics, Nippon Dental University, Niigata, Japan

${ }^{2}$ Department of Dental Materials Science, Nippon Dental University, Niigata, Japan

\begin{abstract}
Magnetic fields can cause human health problems. Low-frequency electromagnetic fields are sometimes induced by electric currents in metallic objects that are worn or used in or on the body, and are unlike the high-frequency electromagnetic fields that produce heat. In low-frequency magnetic fields, the strength of the induced electric current is believed to be more important than the magnetic field strength in terms of effects on the health of living bodies. Electrically powered dental devices, including personal devices such as electric toothbrushes and professional devices such as curing lights for light-cured dental resins, are widely used because of their convenience, but the electric circuits and motors that power them produce low-frequency electromagnetic waves. These magnetic fields induce electric currents in teeth and in metallic appliances that are set in the oral cavity. The effects of these induced currents on human health must therefore be considered. Investigations of this issue revealed that magnetic fields from dental devices promoted the corrosion of intraoral metallic appliances via the induced currents, and this is likely to cause metallic allergies. Almost all reports on the effects of magnetic field exposure, including this corrosion phenomenon, describe harmful effects on human health. However, there may be some effective uses of the magnetic fields generated by dental devices for human health promotion. Oral bacteria were found to corrode orthodontic stainless steel appliances, but magnetic field-induced currents in these appliances could provide remarkable protection effects against this microbially induced corrosion. In this review, we discuss the results and conclusions of these investigations especially about novel ways of harmful effects and effective use of magnetic fields from dental devices, and conclude that further detailed studies are required to clarify their detailed mechanisms and to develop countermeasures for protection from or effective use of these fields.
\end{abstract}

Keywords: Low frequency; Induced current; Corrosion; Electric toothbrushes; Curing lights; Dental appliances; Tooth; Microbiologically influenced corrosion; Protection against corrosion

\section{Introduction}

There has been considerable recent interest in the effects of electromagnetic fields (such as those near electricity transmission lines) on human health, and particularly in their potential to effect the development of leukemia and central nervous system tumors. An elevated risk of leukemia following exposure to electromagnetic fields has been demonstrated [1,2], and the International Agency for Research on Cancer (IARC), the International Commission on Non-Ionizing Radiation Protection (ICNIRP) and the World Health Organization (WHO) have each established guidelines and criteria to outline the roles and risks of magnetic fields (MFs) in carcinogenesis [3-5]. However, other reports in the literature have found no significant correlation between exposure to electromagnetic fields and leukemia [6,7]. The results from studies of the correlation between electromagnetic field exposure and the risks of central nervous system tumors are also inconclusive [2,7]. These findings with regard to the risks of electromagnetic fields inducing leukemia or central nervous system tumors should be considered in terms of dependence on the electromagnetic field exposure conditions, such as field intensity and exposure time [8,9].

Many home electrical appliances, including personal dental devices, generate MFs $[10,11]$. In the dental field, the effects of MFs on human health are thought to be more serious when compared with many other medical fields, because the MF-generating devices are used near the craniocervical area. In a study of dental clinics, electromagnetic fields were detected, and each professional dental device that was set up in the clinics was reported to generate MFs [12]. However, exposure to MFs from professional dental devices for use in dental clinics might be considered to have little effect on the patients, because of the limited times and numbers of clinic visits for each patient. In a questionnairebased survey of the tooth brushing habits of 1200 Japanese people (600 males and 600 females, with an age range from teens to sixties), 52.5\% of the respondents cleaned their teeth twice a day, and $48 \%$ had brushing times of 1-3 min [13]. Based on these figures, the average Japanese person's brushing time was estimated to be $120 \mathrm{~min} / \mathrm{month}(2 \mathrm{~min} \times 2$ times/day $\times 30$ days), and $1440 \mathrm{~min} /$ year. For these reasons, we focused on personal dental devices, and on electric toothbrushes in particular, as the main MF sources. The effects of MF exposure from electric toothbrushes on user health are likely to slowly but surely increase.

In this review, we describe the effects of MFs from electric toothbrushes and other dental devices on dental appliances and teeth that could affect human health. In addition, interesting unpublished data and speculation with regard to MF effects are given in this paper.

\section{MFs from Dental Devices}

Electric toothbrushes are widely used because of their convenience. However, they have also been reported to produce low-frequency

*Corresponding author: Takashi Kameda, Department of Orthodontics, Nippon Dental University, School of Life Dentistry at Niigata, 1-8 Hamaura-cho, Chuo-ku, Niigata 951-8580, Japan, Tel: +81-25-267-1500 ext. 3303; Fax: +81-25-267-1622; E-mail: tkameda@ngt.ndu.ac.jp

Received December 19, 2013; Accepted January 06, 2014; Published January 08,2014

Citation: Kameda T, Ohkuma K (2014) Electromagnetic Fields from Dental Devices and their Effects on Human Health. J Electr Electron Syst 3: 118. doi:10.4172/2332-0796.1000118

Copyright: (c) 2014 Kameda T, et al. This is an open-access article distributed under the terms of the Creative Commons Attribution License, which permits unrestricted use, distribution, and reproduction in any medium, provided the original author and source are credited. 
electromagnetic fields, which could cause health problems, e.g. interference with pacemakers $[10,14]$. In dental clinics, light-cured resins that are polymerized by curing light sources, which have also been reported to produce low-frequency electromagnetic fields, are also widely used for both convenience and speed [11].

The MFs produced by dental devices, e.g. by electric toothbrushes and curing light sources for light-cured resin, and their frequencies were evaluated. We estimated MFs within the $1-2000 \mathrm{~Hz}$ frequency range, in keeping with our preliminary study and with the previous literature, which indicated that this was the appropriate range to monitor $[10,15]$. Five commercially available electric toothbrushes and three commercially available curing lights that were investigated generated MFs [16]. The MFs produced by each representative dental device are shown in Figure 1. The MFs produced by each of the electric toothbrushes were found to be at quite different levels [16]. These MF levels could reflect the cleaning ability of each electric toothbrush. Further investigation of the relationship between MF level and cleaning ability is necessary, and should be useful as part of a design and construction strategy for the development of electric toothbrushes with low MFs and high cleaning ability. Interestingly, the MF levels from curing lights were found to be at low levels in Light-Emitting Diode (LED)-type lights and at high levels in halogen-type lights [17]. Compared with LED-type curing lights, halogen-type curing lights generated much more heat. To prevent these curing lights from overheating, cooling fans with electric motors were built into the halogen-type curing lights. The MF levels of these lights were believed to be dependent on the strengths of the fan electric motors. Similarly, the MFs from electric toothbrushes were found to be at different levels at the front, back, right and left sides of the brushes, which is likely to be related to the location of the motor or other electrical components inside the toothbrushes.

\section{MFs from Dental Devices Induce Electric Currents in Intraoral Metallic Appliances and Teeth}

Many questions have been raised with regard to the effects of MFs from dental devices on human health. There are questions over whether or not these effects are harmful or harmless, and over the mechanisms of MF effects on human health. Many home electrical appliances and professional dental devices generate low-frequency MFs that can induce electric currents in the human body and within any metallic objects or devices worn in or on the body [10,15]. In low-frequency MFs, the strength of the induced electric current is thought to be more important than the strength of the MF itself in terms of the effects on the health of living bodies [18]. To create a framework to discuss these questions, we set up our hypothesis as follows. (1) Dental electrical devices generate MFs (as already described in the previous section) [16,17]. (2) These MFs can induce electric currents in teeth and metallic appliances that are set in the oral cavity [17]. (3) The induced currents produce certain effects that may have an influence on human health. Initially, we investigated part 2 of the hypothesis, e.g. that currents are induced in metallic appliances and teeth by MFs from dental devices (Figure 2). MF exposure was found to induce electric currents in every dental metallic appliance, which were made of various metals, including stainless steel (SUS), CoCr, 70\%Ag alloy, $12 \% \mathrm{Au}-40 \% \mathrm{Ag}$ $20 \%$ Pd alloy, nickel titanium, $\beta$-titanium (Ti-11.5MO-6Zr-4.5Sn) and pure titanium [16]. Interestingly, the measured electric voltages and currents that were induced in the dental appliances were not dependent on the electrical resistivity values of the materials, but on their shapes and specific metal contents [16].

Electric currents were also induced in human hard tissues such as tooth and bone by MFs, and the roles that they play in the living body must be considered, because they may simply cause pain, or may perform roles in bone formation and repair similar to the methods based on electrical or electromagnetic field stimulation (which use MF-induced currents) $[19,20]$. To clarify these roles, currents induced in teeth by MFs from electric toothbrushes and curing lights were investigated. Induced currents were observed in teeth under MF exposure. We found that the voltages induced in teeth by electric toothbrushes were higher $\left(10^{2}-10^{3}\right.$ $\mu \mathrm{V})$ than those previously measured in dental appliances $\left(10^{1}-10^{2} \mu \mathrm{V}\right)$ $[16,17]$. In contrast, the currents induced by electrictoothbrushes in
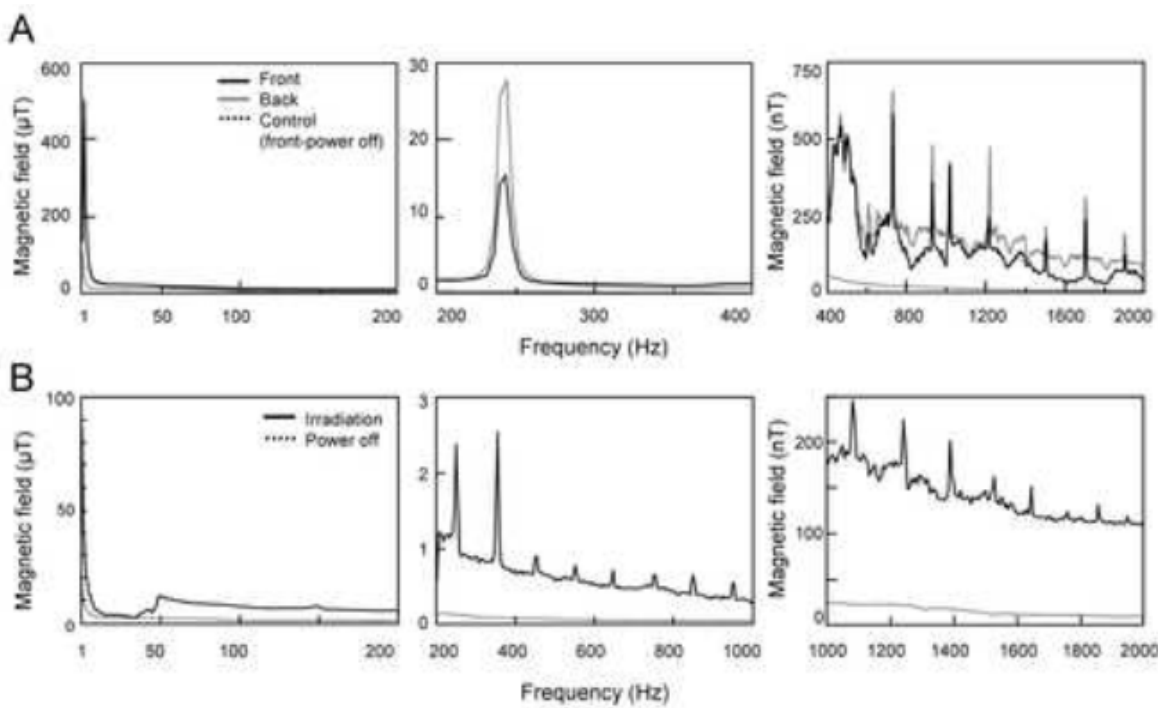

Figure 1: Low-frequency magnetic fields produced by dental devices

A: electric toothbrush (Philips Sonicare HX9100, Philips Oral Healthcare Inc., Bothell, WA); B: curing light (OPTILUX500, Kerr Inc., Camano Island, WA). The MFs produced by the electric toothbrushes and the curing lights, and their frequencies were detected and evaluated using a spectrum analyzer (SPECTRAN NF-5035, Aaronia AB Inc., Euscheid, Germany). The MFs were estimated at $2 \mathrm{~cm}$ distances from the front and back sides of activated or inactive electric toothbrushes, and 1 $\mathrm{cm}$ distances from the light guide tips of activated or inactive curing lights. 


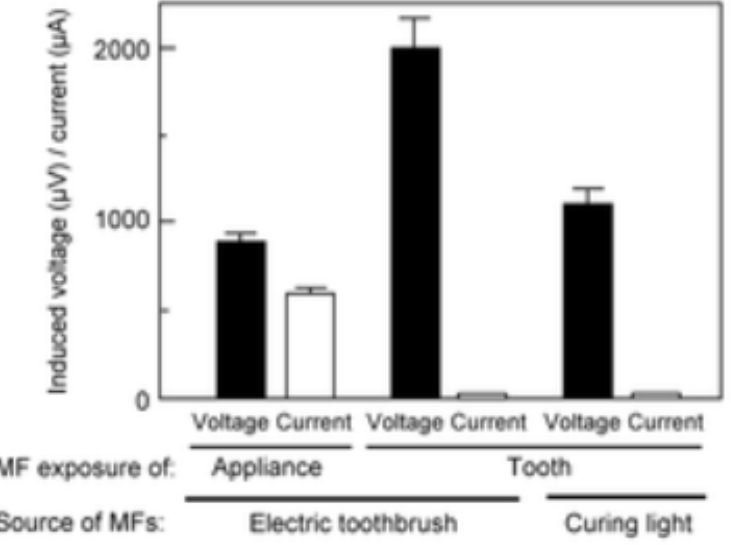

Figure 2: Electric voltages and currents induced in SUS multibracket system and tooth by dental devices at a distance of $1 \mathrm{~cm}$ between device and appliances.

Appliance: multibracket system consisting of brackets (SUS304 $\times 10$ SuperMesh Bracket, Tomy International Inc., Tokyo, Japan), molar tubes (SUS304 $\times 2$, Single Tube, Tomy International Inc.), molar bands (SUS304 $\times 2$, Ideal Molar Band, Tomy International Inc.), and wire (SUS304 × 1 , Suzuki stainless steel wire, Mitsuba Ortho Supply Inc., Tokyo, Japan). Tooth: human maxillary premolar tooth of patient undergoing orthodontic treatment. Electric toothbrush: Philips Sonicare HX9100. Curing light: OPTILUX500. The electric currents induced in dental devices were estimated using a digital multimeter (7351 A/E, ADC Corporation, Tokyo, Japan) in AC+DC mode (i.e. voltage $=\left(\mathrm{ACV}^{2}+\mathrm{DCV} 2\right)^{1 / 2}$, and current $\left.=\left(\mathrm{ACl}^{2}+\mathrm{DCl} 2\right)^{1 / 2}\right)$

\begin{tabular}{|c|c|c|c|c|}
\hline \multirow{2}{*}{} & \multicolumn{2}{|c|}{ Bracket + wire } & \multicolumn{2}{c|}{ Bracket only } \\
\cline { 2 - 5 } & MF (-) & MF (+) & MF (-) & MF (+) \\
\hline $\mathrm{Cr}$ & - & + & - & - \\
\hline $\mathrm{Fe}$ & $0.20 \pm 0.012$ & $1.39 \pm 0.067^{*}$ & $0.11 \pm 0.018$ & $0.71 \pm 0.082^{*}$ \\
\hline $\mathrm{Ni}$ & - & + & - & + \\
\hline $\mathrm{Sn}$ & na & na & - & - \\
\hline $\mathrm{Ti}$ & na & na & - & - \\
\hline
\end{tabular}

$\mathrm{n}=6$ for each experimental condition. SUS: stainless steel wire; MF: magnetic field; + : detected values $(3.3 \delta /$ slope $) \leq$ values $<$ determination limit $\left(10 \times 2^{1 / 2} \delta /\right.$ slope $)$ -: values <detection limit; and na: not available. The data were analyzed via the Mann-Whitney $U$ test to define the statistically significant differences. Superscrip asterisks denote the statistically significant differences $(p<0.05)$ within each appliance compared with *MF(-).

Table 1: Elution amount of each element $(\mu \mathrm{g})$ from SUS appliances to artificial saliva.

teeth were lower $\left(\approx 10^{1} \mu \mathrm{A}\right)$ than those previously measured in dental appliances $\left(10^{1}-10^{3} \mu \mathrm{A}\right)[16]$. This difference could relate to disparities between the electrical resistivities of tooth tissue $\left(\approx 10^{3} \Omega \mathrm{m}\right)$ and metals (e.g. stainless steel $\approx 10^{-8} \Omega \mathrm{m}$ ) [21,22]. We predicted that the ranking order in which currents could be induced in different bracket materials with completely same bracket shape and size (KBT metal bracket; SUS304, KBT zirconia bracket; $\mathrm{ZrO}_{2}$, Rocky Mountain Morita Inc., Tokyo, Japan) would be tooth+stainless steel bracket $>$ tooth+zirconia bracket $\approx$ tooth, based on their electrical resistivities (tooth, $10^{3} \Omega \mathrm{m}$; stainless steel, $10^{-8} \Omega \mathrm{m} ; \mathrm{ZrO}_{2}, 10^{10}-10^{12} \Omega \mathrm{cm}$; resin (acrylic), $10^{13}-10^{14}$ $\Omega \mathrm{m})[23,24]$. However, we found that the actual ranking order was tooth+zirconia bracket $>$ tooth+stainless steel bracket $>$ tooth [17]. The reason for this unexpected result is unclear. The presence of the adhesive does not appear to be a factor, because the induced currents with and without adhesive (no bracket) were comparable. The resistivity of the bracket material also appears to be insignificant, because the induced current was greater in teeth that were attached to $\mathrm{ZrO}_{2}$ or stainless steel brackets than in teeth that were bonded to identically shaped acrylic brackets (unpublished data). Despite considerable efforts, we have been unable to correlate these results with any specific material property. It is likely that this unexpected induced current ranking order is because of a combination of tooth properties and the material properties of the appliance components rather than the materials and their resistivities alone.

In any case, MFs from personal and professional dental devices induced currents, not only in the metallic dental appliances, but also in teeth. The effects of these induced currents on human health must now be considered.

\section{MFs from Dental Devices Promote Corrosion of Intraoral Metallic Appliances via Induced Currents}

Currents induced in intraoral metallic appliances by MFs from dental devices must have some effects. We must therefore determine these effects.

Previous results showed that the MFs from electric toothbrushes could induce alternating electric currents in both dental metallic appliances and teeth [16,17]. Electric currents in oral appliances, e.g. galvanic currents, could cause both discomfort for the user and corrosion of the metallic dental appliances $[25,26]$. Currents induced by electric toothbrushes were also thought to cause some problems in the oral condition of patients. However, the currents induced by the MFs from dental devices are unlike galvanic currents [16,17]. Galvanic currents, which are direct currents, produced high voltages (several tens to hundreds of $\mathrm{mV}$ ) with low currents (several tens to hundreds of $\mathrm{nA}$ ), whereas the electric currents that were induced by electric toothbrushes were of the order of $\mu \mathrm{A}$ to $\mathrm{mA}$ with a voltage range of $\mu \mathrm{V}$ to $\mathrm{mV}$ [16]. These findings seemed to indicate that galvanic currents were one of the causes of metal corrosion, whereas the electric currents that were induced in dental appliances should not directly cause metal corrosion. However, there were some reports in the literature about metallic corrosion caused by induced Alternating Currents (AC). Rapid geomagnetic variations have been known to induce electric currents in power lines and pipelines, which could then lead to the destruction of power transmission systems and pipeline corrosion [27]. In the straycurrent corrosion caused by alternating currents, the induced currents occurred in embedded metal objects lying parallel to the high voltage $\mathrm{AC}$ power lines or to the transportation routes of AC electric railways, which then caused the corrosion of the embedded metal objects [28]. Based on these reports, we must therefore consider that the induced currents in metallic appliances produced by MFs from dental devices could be a trigger for corrosion in metallic appliances.

Metallic elution and the surface roughness of metallic appliances immersed in artificial saliva during MF exposure were investigated using a ICP-OES (iCAP 6300 Duo, Thermo Fisher Scientific, Waltham, MA, USA) and a 3D laser confocal microscope (LEXT OLS4000, Olympus Inc., Tokyo, Japan) [29]. MF exposure (120 min; $24 \mathrm{~min} /$ day $\times 5$ days) dramatically increased Fe elution, with low levels of $\mathrm{Cr}$ and Ni elution from SUS appliances (Table 1). The surface roughness of SUS appliances after MF exposure supported these results (Figures 3 and 4). In contrast to SUS appliances, Ti elution and increased surface roughness were not observed in titanium appliances [29]. In addition, while the induced voltages were almost identical in SUS and titanium appliances, the induced currents in titanium appliances were double those in SUS appliances [29]. These values were not in proportion to the material electrical resistivities, which were 70-80 $\Omega \mathrm{m}$ for SUS304 and $80-100 \Omega \mathrm{m}$ for NiTi. In contrast to the surface roughness evidence 
Citation: Kameda T, Ohkuma K (2014) Electromagnetic Fields from Dental Devices and their Effects on Human Health. J Electr Electron Syst 3: 118. doi:10.4172/2332-0796.1000118

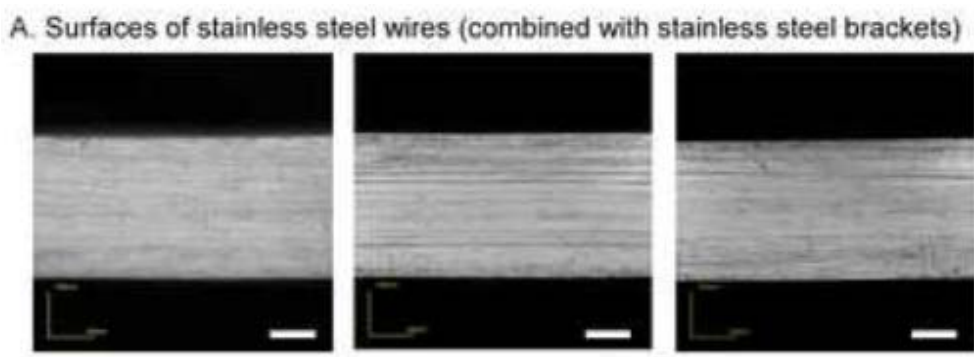

B. Surfaces of stainless steel brackets (combined with stainless steel wires)

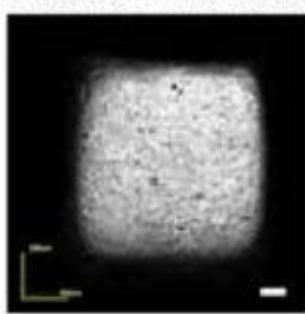

No treatmen:

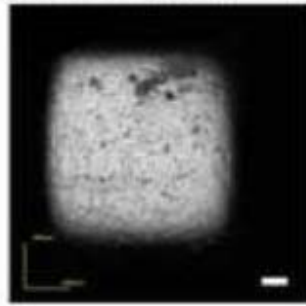

Immersed in artificial saliva

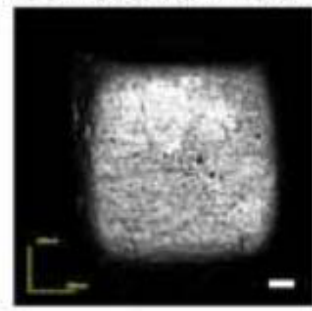

Exposure to magnetic field in artificial saliva

Figure 3: 3D laser confocal micrographs of SUS wire and bracket surfaces immersed in artificial saliva with or without exposure to MFs from electric toothbrushes (×20). The white lines in the micrographs represent $100 \mu \mathrm{m}$.
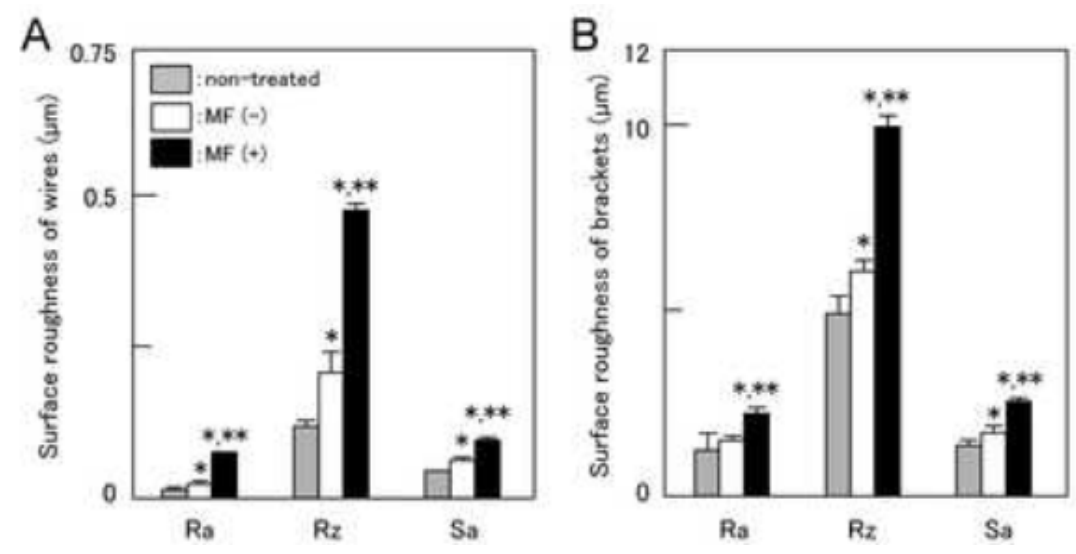

Figure 4: Surface roughnesses of SUS brackets and SUS wires immersed in artificial saliva with or without exposure to MFs from electric toothbrushes.

$n=6$ for each experimental condition. Ra represents the arithmetic average of the absolute value $\left(R a=n^{-1} \Sigma|Y i|(i=1-n)\right.$ ), Rz represents the highest peaks and lowest valleys over the entire sampling length, and Sa represents the arithmetic average of the 3D roughness (areal roughness). The data were analyzed via the MannWhitney $U$ test to define the statistically significant differences. Asterisks denote the statistically significant differences $(p<0.05)$ within each measurement item of each appliance group when compared with *non-treated and **MF(-).

for the occurrence of metallic elution, no $\mathrm{pH}$ differences were observed between the non-MF-exposed and MF-exposed groups measured with a $\mathrm{pH}$ meter (F-12, Horiba Inc., Kyoto, Japan) [29]. The $\mathrm{pH}$ values were lowered by the immersion of the appliance in each case; however, these $\mathrm{pH}$ values, which did not reach the depassivation $\mathrm{pH}$, could not have led to the erosion of these alloys [30,31]. However, these $\mathrm{pH}$ values in the immersed solutions may not always reflect the local $\mathrm{pH}$ values around the appliances.

In any case, these results suggested the following novel MF phenomena for human health. (1) The currents induced in metallic appliances by MFs from dental devices could be one of the causes of SUS corrosion. (2) The corrosion resistivity of titanium alloys is high compared with that of SUS alloys. (3) The $\mathrm{pH}$ values of the solutions used for immersion, with or without MF exposure, showed no statistical significance. The low-frequency MFs around the oral cavity that originate from electronic equipment such as electric toothbrushes induce electrical currents in intraorally installed metals (especially in SUS), prostheses and appliances, and promote metallic elution, which is likely to be one of the causes of metallic allergies.

\section{What are the Possible Effects of MFs from Dental Devices on Human Health?}

The corrosion of metallic appliances under MF exposure could have detrimental results for human health, such as metal allergies. The induced current itself could also produce similar results, e.g. pain and discomfort for the patients. Because the role of low- 
frequency electromagnetic fields in carcinogenesis, and especially in the development of leukemia and central nervous system tumors, has been well-documented [1,2,6-9], the effects of MFs were believed to be basically harmful to human health. It is very difficult to protect ourselves from low-frequency MFs because they can pass through human tissue and most other materials, including glass, plastics, metals and concrete [32]. The only viable way to limit our exposure to lowfrequency electromagnetic fields is thought to be to eliminate their generation by electrical home appliances and dental devices.

However, there may also be some effective positive use of MFs generated by dental devices for human health. First, we considered that the induced current in appliances could be used for biocidal applications or for suppression of dental caries- or periodontal diseasecausing microorganisms. However, the number of oral bacteria were not reduced, but were actually increased by the electric currents in appliances under MF exposure from dental devices (unpublished data). The oral bacteria suppression effects might not be caused by MF exposure.

The intraoral aging of dental materials includes the plasticization of polymeric adhesives and increased porosity and roughness in metallic alloys, which disturbs the smooth progress in courses of treatment and causes losses in terms of time and money, along with emotional distress for both patients and clinicians [33,34]. The oral environments of living organisms, including those of humans, are most hospitable for biofilm creation and biocorrosion (microbially influenced corrosion or MIC). If MIC existed in oral areas, the currents induced in metallic appliances by MF exposure can be considered a means of protection against corrosion, like electrical protection in other fields. We first investigated the existence of MIC based on oral bacteria. Three representative indigenous oral bacteria, Streptococcus mutans, Streptococcus sanguinis and Aggregatibacter actinomycetemcomitans, were selected for this experiment. S. mutans and S. sanguinis were oral bacteria that easily created biofilms on the surfaces of the teeth and appliances. A. actinomycetemcomitans is a pathogenic bacterium that occurs in localized aggressive periodontitis. We found that $S$. mutans (ATCC49296) and S. sanguinis (ATCC25175) corroded SUS dental appliances when the appliances were cultured with them in BHI medium (Brain Heart Infusion medium, Beckton, Dikinson and Company Inc., Detroit, MI, USA) at $37^{\circ} \mathrm{C}$ in $5 \% \mathrm{CO} 2$ in air in the incubators without shaking from the results of metallic elution and surface roughness of appliances by a ICP-OES and a 3D laser confocal microscope respectively [35], but A. actinomycetemcomitans did not corrode SUS or titanium appliances (unpublished data). We then investigated the protection effects of MF-exposure against the biocorrosion of these appliances at a distance of $2 \mathrm{~cm}$ between device and appliances. In the groups that were co-cultured with $S$. sanguinis, remarkable protection effects of $120 \mathrm{~min}(24 \mathrm{~min} /$ day $\times 5$ days $)-\mathrm{MF}$ exposure against MIC were observed (Figure 5). This MF effect on oral bacterial corrosion of metallic appliances is novel effective use of MFs from dental devices. However, the reasons for this result are unclear, and further detailed investigation is required.

In the orthopedic field, MFs have been used for bone repair in methods based on electrical or electromagnetic field stimulation $[19,20]$. The MFs from dental devices were found to induce currents in human hard tissue, e.g. teeth [17]. These results indicate the possibility that MFs from dental devices could induce electric currents not only in teeth but also in bone tissue, which would then promote bone formation and repair.

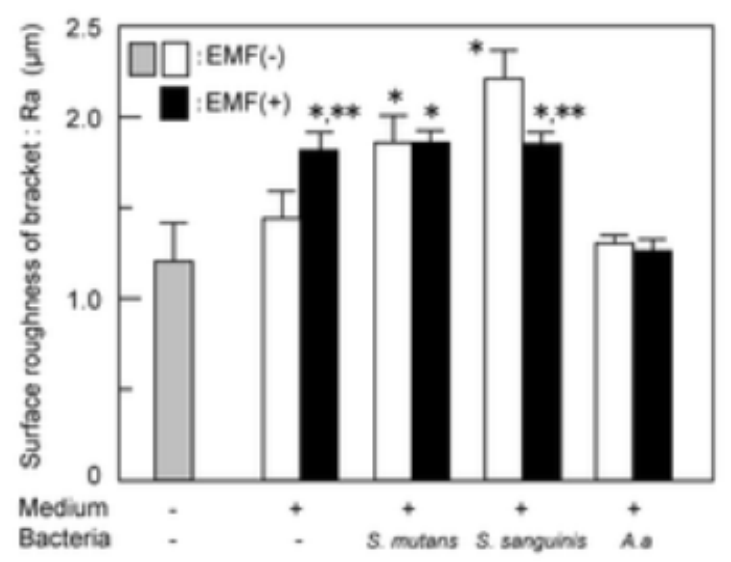

Figure 5: Surface roughnesses of SUS brackets immersed and co-cultured with oral bacteria and with or without exposure to MFs from electric toothbrushes.

$\mathrm{n}=6$ for each experimental condition. Ra represents the arithmetic average of the absolute value. A. a: Aggregatibacter actinomycetemcomitans. The data were analyzed via the Mann-Whitney $U$ test to define the statistically significant differences. Asterisks denote the statistically significant differences $(p<0.05)$ compared with *intact products (medium (-) and bacteria (-) group) and within each measurement item of each group

when compared with ${ }^{* *} \mathrm{MF}(-)$

\section{Conclusions and Outlook}

This review has discussed the electromagnetic fields of dental devices and their effects on human health. The electromagnetic fields produced by dental devices were basically low-frequency MFs. MFs have been considered to be generally harmful to human health, especially in the development of leukemia and central nervous system tumors $[1,2,6-9]$. However, it is very difficult to protect ourselves from these low-frequency MFs, because they easily pass through human tissue and most other materials [32].

Under these conditions, there are two possible conclusions as to what can be done about MFs from dental devices:

1. Find ways to use MFs from dental devices effectively for human health promotion;

2. Abolish the devices that generate these MFs, or reduce the MF levels generated bythe dental devices.

The first conclusion was investigated and discussed in the sections above, eg. suppression of oral bacterial corrosion of intraoral dental appliances, and promotion of bone formation and repair. Further and more detailed study is needed to establish effective methods for MF utilization. With regard to the second conclusion, viable way to limit exposure to low-frequency electromagnetic fields is thought to be elimination of their generation by dental devices, eg. development of dental devices with low MF generation and keeping the devices away from tooth and metallic appliances as possible, and/or elimination of metallic appliances from oral cavity. New regulatory standards specific to human health, and particularly for the craniocervical region, are also needed in conjunction with the current conventional EMC (electromagnetic compatibility) standards, such as ISO standards.

In conclusion, further study is required to clarify the mechanisms by which the electric currents that are induced by MFs affect human oral health and to explore whether countermeasures can be developed to provide methods of protection from or methods for effective use of these effects. 
Citation: Kameda T, Ohkuma K (2014) Electromagnetic Fields from Dental Devices and their Effects on Human Health. J Electr Electron Syst 3: 118. doi:10.4172/2332-0796.1000118

\section{Acknowledgement}

The authors would like to thank Assistant Prof. Hirotake Oda (Department of Periodontology, Nippon Dental University, Niigata) for his technical assistance with bacterial cultures, and Prof. Soh Sato (Department of Periodontology, Nippon Dental University, Niigata) and Prof. Kazuto Terada (Department of Orthodontics Nippon Dental University, Niigata) for providing valuable advice.

\section{References}

1. Ahlbom A, Day N, Feychting M, Roman E, Skinner J, et al. (2000) A pooled analysis of magnetic fields and childhood leukaemia. Br J Cancer 83: 692-698.

2. Floderus B, Persson T, Stenlund C, Wennberg A, Öst $\AA$, et al. (1993) Occupational exposure to electromagnetic fields in relation to leukemia and brain tumors: a case-control study in Sweden. Cancer Causes Control 4: 465476

3. International Agency for Research on Cancer (2002) IARC monographs on the evaluation of carcinogenic risks to humans, $80 \mathrm{pt} 1$.

4. International Commission on Non-lonizing Radiation Protection (1998) Guide lines for exposure to time-varying electric, magnetic, and electromagnetic fields (up to $300 \mathrm{GHz}$ ).

5. World Health Organization (1987) Environmental health criteria 69: Magnetic fields. Geneva, Switzerland.

6. Thériault G, Goldberg M, Miller AB, Armstrong B, Guénel P, et al. (1994) Cance risks associated with occupational exposure to magnetic fields among electric utility workers in Ontario and Quebec, Canada, and France: 1970-1989. Am J Epidemiol 139: 550-572.

7. Savitz DA, Loomis DP (1995) Magnetic field exposure in relation to leukemia and brain cancer mortality among electric utility workers. Am J Epidemiol 141: 123-134.

8. Kheifets L, Ahlbom A, Crespi CM, Draper G, Hagihara J, et al. (2010) Pooled analysis of recent studies on magnetic fields and childhood leukaemia. $\mathrm{Br} J$ Cancer 103: 1128-1135.

9. Kheifets L, Ahlbom A, Crespi CM, Feychting M, Johansen C, et al. (2010) A pooled analysis of extremely low-frequency magnetic fields and childhood brain tumors. Am J Epidemiol 172: 752-761.

10. http://www.aeha.or.jp/report/

11. Seki K, Sugano N, Nanba, K, Moriya, Y, Orii, H et al. (2003) Measurement of electromagnetic waves from dental devices. Nihon Univ Dent J 77: 359-361.

12. Kanda N, Kawabe S, Ikeda T, Takeyama M (2001) Electromagnetic wave enviromental measurement in the medical facilities -The measurement example of the dental hospital, Kenchiku Zasshi 116 suppl: 505-506.

13. http://research.lifemedia.jp/2011/06/1110601_teeth.html

14. http://www.intechopen.com/books/modern-pacemakers-present-andfuture/ electromagnetic-interference-of-the-pacemakers

15. Kamimura Y, Yamada Y, Akutsu T (2005) Induced current inside the human head in the vicinity of an electric shaver. EMC J 104: 61-64.

16. Kameda T, Ohkuma K, Ishii N, Sano N, Ogura H, et al. (2012) Electric toothbrushes induce electric current in fixed dental appliances by creating magnetic fields. Dent Mater J 31: 856-862.

17. Kameda T, Ohkuma K, Sano N, Ogura H, Terada K (2013) Electric current induced in teeth by electromagnetic fields from electric toothbrushes and curing lights. Orthod Waves 72: 77-85

18. Miyakoshi J, Yamagishi N, Ohtsu S, Mohri K, Tanabe H (1996) Increase in hypoxanthine-guanine phosphoribosyl transferase gene mutation by exposure to high density 50-Hz magnetic fields. Mutat Res 349: 109-114.

19. Jahn TL (1968) A possible mechanism for the effect of electrical potentials on apatite formation in bone. Clin Orthop 56: 261-273.

20. Bassett CA, Mitchell SN, Gaston SR (1981) Treatment of ununited tibia diaphyseal fractures with pulsing electromagnetic fields. J Bone Joint Surg 63 511-523.

21. Schulte, A, Gente, M, Pieper, K, Arends, J (1998) The electrical resistance of enamel-dentine cylinders. Influence of $\mathrm{NaCl}$ content in storage solutions. J Dent 26: 113-118.

22. Lide RD (1995) Handbook of Chemistry and of Physics $\left(75^{\text {th }}\right.$ edn.). The Chemical Rubber Co, London, UK.

23. Ortiz A, Alonso JC, Haro-Poniatowski E (2006) Spray deposition and characterization of zirconium-oxide thin films. J Electron Mater 34: 150-155.

24. Yu Y-H, Ma CC, Yuen SM, Teng CC, Huang YL, et al. (2010) Morphology, electrical, and rheological properties of silane-modified silver nanowire/polymer composites. Macromol Mate Eng 295: 1017-1024.

25. Karov J, Hinberg I (2001) Galvanic corrosion of selected dental alloys. J Oral Rehabil 28: 212-219.

26. Bakhtari A, Bradley TG, Lobb WK, Berzins DW (2011) Galvanic corrosion between various combinations of orthodontic brackets and archwires. Am J Orthod Dentofacial Orthop 140: 25-31.

27. Nagatsuma T (2002) Geomagnetic storms. J Commun Res Lab, 48: 123-136.

28. Fujii $T$ (2011) Metal corrosion ( $1^{\text {st }}$ edn.). Business \& Technology Daily News Inc., P64.

29. Kameda T, Ohkuma K, Oda H, Sano N, Batbayar N, et al. (2013) Magnetic fields from electric toothbrushes promote corrosion in orthodontic stainless steel appliances but not in titanium appliances. Dent Mater J 32: 959-969.

30. Nash BK; Kelly RG (1993) Characterization of the crevice solution chemistry of 304 stainless steel. Corrosion Sci 35: 817-825

31. Schiff N., Grosgogeat B, Lissac M, Dalard F (2002) Influence of fluoride content and $\mathrm{pH}$ on the corrosion resistance of titanium and its alloys. Biomater 23 : 1995-2002.

32. http://www.cancer.gov/cancertopics/factsheet/Risk/magnetic-fields

33. Eliades T, Athanasios AE (2002) In vivo aging of orthodontic alloys: Implications for corrosion potential, nickel release, and biocompatibility. Angle Orthod 72 222-237.

34. Eliades T, Bourauel C (2005) Intraoral aging of orthodontic materials: the picture we miss and its clinical relevance. Am J Orthod Dentfacial Orthop 127 403-412.

35. Kameda T, Oda H, Ohkuma K, Sano N, Batbayar N, et al. (2014) Microbiologically influenced corrosion of orthodontic metallic appliances. Dent Mater J 33: in press
This article was originally published in a special issue, Electromagnetic Wave

Theory handled by Editor(s). Dr. Cheng-Wei Qiu, National University of Sin-

gapore, Singapore. 